PROCEEDINGS OF THE

AMERICAN MATHEMATICAL SOCIETY

Volume 136, Number 9, September 2008, Pages 3007-3011

S 0002-9939(08)09245-9

Article electronically published on April 23, 2008

\title{
A NOTE ON THE JACOBIAN CONJECTURE
}

\author{
CHRISTOPHER I. BYRNES AND ANDERS LINDQUIST
}

(Communicated by Paul Goerss)

\begin{abstract}
In this paper we consider the Jacobian conjecture for a map $f$ of complex affine spaces of dimension $n$. It is well known that if $f$ is proper, then the conjecture will hold. Using topological arguments, specifically Smith theory, we show that the conjecture holds if and only if $f$ is proper onto its image.
\end{abstract}

\section{InTRODUCTION}

Consider the field $\mathbb{C}$ and a polynomial mapping $f: \mathbb{C}^{n} \rightarrow \mathbb{C}^{n}$ such that the Jacobian, $\operatorname{det} \operatorname{Jac}(f)$, is a nonzero constant. Formulated by O. H. Keller in 1939 [8], the Jacobian conjecture is that $f$ must have a polynomial inverse. It will be convenient, however, to express existence of an inverse in more modern terms, which we briefly review. In order to keep domains, ranges and images straight, we express any polynomial map $g: \mathbb{C}^{n} \rightarrow \mathbb{C}^{n}$ as a map

$$
g: X \rightarrow Y
$$

where $X$ and $Y$ are complex affine $n$-spaces. We call such a map regular. Note that each of its components is a polynomial map from $X$ to $\mathbb{C}$. We denote the ring of all such maps by $\mathbb{C}[X]$ and refer to its elements as regular.

In particular, any regular map (10) induces a homomorphism of rings

$$
g^{*}: \mathbb{C}[Y] \rightarrow \mathbb{C}[X]
$$

where $g^{*} h=h \circ g$. To say that $g$ has a regular inverse is therefore to say that $g^{*} \mathbb{C}[Y]=\mathbb{C}[X]$, and we will call such a map biregular. We denote by $\mathbb{C}(X)$ and $\mathbb{C}(Y)$ the fields of rational functions on $X$ and $Y$, respectively. A weaker property than biregularity is the property that $g$ be birational, i.e. that $g^{*} \mathbb{C}(Y)=\mathbb{C}(X)$.

We refer to the excellent survey by Bass, Connell and Wright [2] for more historical and technical details. In particular, they list eight conditions, including the conclusion of the Jacobian conjecture, which for algebraically closed fields in characteristic zero are equivalent. Our interest is in four of these for the field $\mathbb{C}$ and for the map $f$ :

(i) $f$ is biregular,

(ii) $f$ is birational,

Received by the editors October 25, 2006.

2000 Mathematics Subject Classification. Primary 14R15, 55M35; Secondary 47H10.

Key words and phrases. Jacobian conjecture, Smith theory.

This research was supported in part by grants from AFOSR, NSF, the Swedish Research Council, and the Göran Gustafsson Foundation.

(C) 2008 American Mathematical Society Reverts to public domain 28 years from publication 
(iii) $f$ is injective,

(iv) $f$ is proper.

To say that a continuous map $g: X_{1} \rightarrow X_{2}$ is proper is to say $g^{-1}(K)$ is compact in $X_{1}$ for each compact subset $K \subset X_{2}$. Our main result, Theorem 1 shows that relaxing the last condition still gives a necessary and sufficient condition for the Jacobian conjecture to hold.

\section{The MAIN RESUlT}

Theorem 1. Suppose that $f: X \rightarrow Y$ is a regular map having a constant nonzero Jacobian. Then $f$ is biregular if and only if $f: X \rightarrow f(X)$ is proper.

Proof. If $f$ is biregular, then $f$ is a homeomorphism onto $Y=f(X)$ and is therefore proper onto $f(X)$.

Conversely, since $\operatorname{det} \operatorname{Jac}(f)$ is a nonzero constant, from the inverse function theorem it follows that $f(X)$ is open in $Y$ in the classical topology. In particular, both $X$ and $f(X)$ are smooth, connected, oriented manifolds of real dimension $2 n$. For reasons that will become clear quite soon, we want to distinguish between the map $f$ between complex affine spaces and the map

$$
f_{\mathbb{R}}: X \rightarrow f(X)
$$

of real manifolds. Since (2) is proper and since each $y \in f(X)$ is a regular value, the set $f^{-1}(y)$ is always finite for each $y$. Moreover, this map has a well-defined degree [4, pp. 40-41 and p. 47], $\operatorname{deg}\left(f_{\mathbb{R}}\right)$, which satisfies

$$
\operatorname{deg}\left(f_{\mathbb{R}}\right)=\sum_{x \in f^{-1}(y)} \operatorname{sign} \operatorname{det} \operatorname{Jac}_{x}\left(f_{\mathbb{R}}\right)
$$

for every regular value $y \in f(X)$, which in our setting is for every $y \in f(X)$.

We will begin our proof by showing that (2) defines a covering space. We first note that $X$ and $f(X)$ are connected, locally path-connected spaces, to which the theory of covering spaces [6] does apply. While we make no assumptions about $G=\pi_{1}(f(X))$, the fundamental group of $f(X)$, the space $X$ is an affine Euclidean space and is therefore simply connected. It will turn out that $G$ is finite and acts freely on $X$; i.e., $g x=x$ for some $x \in X$ implies $g=e$, where $e$ is the identity element in $G$.

Lemma 1. The map (2) exhibits $X$ as a universal covering space for $f(X)$, for which

$$
d=\# f^{-1}(y)=\operatorname{deg}\left(f_{\mathbb{R}}\right)
$$

is finite. In particular, $G$ has order $d$ and acts continuously and freely on $X$ as the group of covering transformations.

Proof. We first compute $\operatorname{deg}\left(f_{\mathbb{R}}\right)$ using (3). To this end, note that if $T: \mathbb{C}^{n} \rightarrow \mathbb{C}^{n}$ is $\mathbb{C}$-linear, then $T$ induces an $\mathbb{R}$-linear map

$$
T_{\mathbb{R}}: \mathbb{R}^{2 n} \rightarrow \mathbb{R}^{2 n}
$$

which satisfies $\operatorname{det} T_{\mathbb{R}}=|\operatorname{det} T|^{2}$. Applying this observation to $T=\operatorname{Jac}_{x}(f)$, we see that

$$
\operatorname{deg}\left(f_{\mathbb{R}}\right)=\sum_{x \in f^{-1}(y)} \operatorname{sign}\left|\operatorname{det} \operatorname{Jac}_{x}(f)\right|^{2}=\# f^{-1}(y)
$$


for each $y \in f(X)$. We denote the common integer value in this equation by $d$.

From this it is easy to deduce that (2) exhibits $X$ as a covering space for $f(X)$. For each $y \in f(X)$, we have $f^{-1}(y)=\left\{x_{1}, \ldots, x_{d}\right\}$, and, for each $i=1, \ldots, d$, there exist open neighborhoods $U_{i}$ of $x_{i}$ and $V_{i}$ of $y$ such that $U_{1}, \ldots, U_{d}$ are pairwise disjoint and $f: U_{i} \rightarrow V_{i}$ is a diffeomorphism for each $i$. Setting $V=\bigcap_{i=1}^{d} V_{i}$, choose open subsets $W_{i} \subset U_{i}$ such that $f: W_{i} \rightarrow V$ is a diffeomorphism and consider the open set $U=f^{-1}(V)$. It is clear that $\bigcup_{i=1}^{d} W_{i} \subset U$, while the reverse inclusion follows from the constancy of the cardinality of the fiber $f^{-1}(y)$.

Therefore, (2) defines a covering space and, since $X$ is simply connected, we have a universal covering space

$$
f_{\mathbb{R}}: X \rightarrow f(X) .
$$

In particular, it follows from [6. Theorem 5.8] that $G$ is canonically isomorphic to the group of covering transformations, that for each $y \in f(X)$ there is a natural bijection between $G$ and the fiber $f_{\mathbb{R}}^{-1}(y)[\underline{6}$, Corollary 5.6], and therefore that $G$ acts freely on each fiber. This concludes the proof of the lemma.

Lemma 2. The map $f$ is injective.

Proof. To say that $d>1$ is to say that there exists a prime number $p$ dividing $d$. By Cauchy's theorem [7, p. 74], $G$ contains an element of order $p$, which generates a cyclic subgroup. This leads to fixed point free action of $\mathbb{Z}_{p}$ on $\mathbb{R}^{2 n}$, contrary to one of the corollaries of Smith theory. More explicitly, the continuous action of $\mathbb{Z}_{p}$ extends to a continuous action on $S^{2 n}$, leaving the north pole fixed. The main theorem of Smith theory for spheres [10] is that the fixed point set $F$ in $S^{2 n}$ is a homology $\ell$-sphere, with coefficients in $\mathbb{Z}_{p}$, with $\ell=-1,0,1, \ldots, 2 n$. Here, $\ell=-1$ denotes the empty set, a situation that cannot occur in the present setting, since the north pole is fixed. In particular, for $\ell \geq 0, f$ has at least two fixed points, and at least one of these must lie in $X$, which contradicts the assumption that we have a fixed point free $\mathbb{Z}_{p}$ action on $X$. Therefore $d=1$.

Lemma 3. The map $f$ is a diffeomorphism of $X$ onto $Y$.

Proof. By the theorem of Bialynicki-Birula and Rosenlicht [3, an injective polynomial map of affine spaces of the same dimension is surjective, so that $f$ is a bijection of $X$ with $Y$. By the inverse function theorem, $f^{-1}$ is differentiable, so that $f$ is a diffeomorphism.

Lemma 4. The map $f: X \rightarrow Y$ is birational.

Proof. Since $f: X \rightarrow Y$ is a regular surjection, $f^{*}$ maps $\mathbb{C}(Y)$ injectively to $\mathbb{C}(X)$, and so $\mathbb{C}(X)$ may be regarded as a vector space over the field $f^{*} \mathbb{C}(Y)$. Since $\operatorname{dim}(X)=\operatorname{dim}(Y)$, this vector space is finite dimensional, and, according to 9 , p. 46], we have

$$
\# f^{-1}(y)=\operatorname{dim}_{f * \mathbb{C}(Y)} \mathbb{C}(X)
$$

for $y \in Y$ a regular value for $f$. On the other hand, all points $y \in Y$ are regular values, and, by Lemma 2. the left-hand side of (6) equals 1 . Therefore, $f^{*} \mathbb{C}(Y)=$ $\mathbb{C}(X)$.

Lemma 5. The map $f: X \rightarrow Y$ is biregular. 
Proof. We have shown that the map $f$ is a regular bijection, between the smooth affine varieties $X$ and $Y$. Therefore, by Zariski's main theorem [9, p. 48], $f$ is biregular.

This concludes the proof of the theorem.

\section{Concluding REMARKS}

In this paper, we have introduced to the original form of the Jacobian conjecture an auxiliary hypothesis, which is both necessary and sufficient for the conclusion of the conjecture to hold. It is therefore logically equivalent to the conditions (i) -(iv) enumerated in Section 1, although it is formally weaker than condition (iv) and seems to be easier to check in principle. It is also interesting to note that our method of proof incorporated two beautiful tools - Smith theory [10] and the theorem of Bialynicki-Birula and Rosenlicht [3] - which have not been used, to the best of our knowledge, in the prior literature on this conjecture.

In fact, using the arguments in our proof, we can give a new proof of the equivalence of (i)-(iv). Of course, (i) trivially implies (iv). Continuing in this cycle, (iv) certainly implies that $f: X \rightarrow f(X)$ is proper. From this, (iii) follows using Lemmas 1 and 2. That (iii) implies (ii) is the content of Lemmas 3 and 4 Finally, that (ii) implies (i) requires a proof (using a well-known argument) that the birational map $f$ is a bijection. To say that $f$ is injective is to say that $f^{-1}(y)$ has at most one point for each $y \in Y$, so we may assume that $y \in f(X)$. For a Zariski open $W \subset f(X)$, we have

$$
\# f^{-1}(y)=\operatorname{dim}_{f * \mathbb{C}(Y)} \mathbb{C}(X)=1
$$

for each $y \in W$, since $f$ is birational. For $y_{0} \in f(X) \backslash W$, suppose $f^{-1}\left(y_{0}\right)=$ $\left\{x_{1}, \ldots, x_{r}\right\}$. There exist open, pairwise disjoint neighborhoods $U_{i}$ of $x_{i}$ and open neighborhoods of $V_{i}$ of $y_{0}$, for $i=1, \ldots, r$, such that $f: U_{i} \rightarrow V_{i}$ is a diffeomorphism for each $i$. Set $V=\bigcap_{i=1}^{r} V_{i}$. If $y_{j} \in W$ is a sequence converging to $y_{0}$, then ultimately $y_{j} \in W \cap V$ for infinitely many $j$ and therefore $r \leq \# f^{-1}\left(y_{j}\right)=1$. Thus, $f$ is injective. By the theorem of Bialynicki-Birula and Rosenlicht, $f$ is surjective, and (i) then follows from Zariski's main theorem.

Remark 1 (Connection with Galois theory). The paper 2 lists several other conditions which are equivalent to (i)-(iv), including the assumption that $\mathbb{C}(X)$ is a normal extension of $f^{*} \mathbb{C}(Y)$. This extension is therefore a Galois extension with a finite group $\mathcal{G}$ acting linearly on $\mathbb{C}(X)$ with fixed subfield $f^{*} \mathbb{C}(Y)$. Indeed the order of $\mathcal{G}$ is given by

$$
\# \mathcal{G}=\operatorname{dim}_{f * \mathbb{C}(Y)} \mathbb{C}(X) .
$$

On the other hand, we assumed that the map $f: X \rightarrow f(X)$ is proper and showed that it defines a universal covering space, which is therefore a normal covering with covering group $G$ having order $\# \mathcal{G}$.

This is not coincidental. Indeed, the proof in [5] that normality and the Jacobian hypothesis imply the Jacobian conjecture is in fact based on a realization of $\mathcal{G}$ as the group $G$ of covering transformations (see 1 for an earlier treatment of the case $n=2$ ). This, however, is where our proof and that in 5 diverge. Campbell proves his theorem by arguing that the base space of the covering is simply connected, as the complement in the simply connected space $\mathbb{C}^{n}$ of an analytic set of codimension 
at least two. Our proof is quite different and follows instead from properties of the total space of the covering, viz. contractibility and its consequences in Smith theory.

Remark 2 (The real case). Our proof yields positive results for the real Jacobian conjecture as well. More explicitly, suppose $f: \mathbb{R}^{n} \rightarrow \mathbb{R}^{n}$ is a polynomial map with det $\operatorname{Jac}(f)$ everywhere positive. Then, Lemmas 11 and 2 apply, mutatis mutandis. Indeed, the first use of algebraic geometry arises in the proof of Lemma 3 through the application of the theorem of Bialynicki-Birula and Rosenlicht - which was stated and proved in 3. for real polynomial maps.

In particular, by combining Lemmas 1 3. we obtain a version of Theorem 1 over $\mathbb{R}$, viz., a real polynomial map $f: \mathbb{R}^{n} \rightarrow \mathbb{R}^{n}$ having a nowhere zero Jacobian is a diffeomorphism onto $\mathbb{R}^{n}$ if, and only if, $f: \mathbb{R}^{n} \rightarrow f\left(\mathbb{R}^{n}\right)$ is proper. This is a sharp result, as the example $f(x)=x^{3}+x$ shows, in that the inverse need not be a polynomial. In this direction, algebraic geometry over $\mathbb{C}$ is used heavily in the proofs of Lemmas 4 and 5 and the weak Jacobian conjecture asks whether, in addition, their combined conclusion would hold (over $\mathbb{R}$ ) whenever the necessary condition, $\operatorname{det} \operatorname{Jac}(f)=c \neq 0$, is satisfied.

\section{REFERENCES}

[1] S. S. Abyhankar, Expansion techniques in algebraic geometry, Tata Inst. Fundamental Research, Bombay, 1977.

[2] H. Bass, E. H. Connell and D. Wright, The Jacobian conjecture: Reduction of degree and formal expansion of the inverse, Bull. Amer. Math. Soc. (N.S.) 7 (1982), 287-330. MR663785 (83k:14028)

[3] A. Bialynicki-Birula and M. Rosenlicht, Injective morphisms of real algebraic varieties, Proc. Amer. Math. Soc. 13 (1962), 200-203. MR0140516 (25:3936)

[4] R. Bott and L. W. Tu, Differential Forms in Algebraic Topology, Springer-Verlag, New York, Heidelberg, Berlin, 1982. MR658304 (83i:57016)

[5] L. A. Campbell, A condition for a polynomial map to be invertible, Math. Ann. 205 (1973), 243-248. MR0324062 (48:2414)

[6] M. Greenberg, Lectures on Algebraic Topology, W. A. Benjamin, Inc., New York, 1967. MR0215295 (35:6137)

[7] I. N. Herstein, Topics in Algebra, Blaisdell Publishing Co., Ginn and Co., New York, Toronto, London, 1964. MR0171801 (30:2028)

[8] O. H. Keller, Ganze Cremona-Transformationen, Monats. Math. Physik 47 (1939), 299-306. MR 1550818

[9] D. Mumford, Algebraic Geometry I: Complex Projective Varieties, Springer-Verlag, Berlin, Heidelberg, New York, 1976. MR0453732 (56:11992)

[10] P. A. Smith, Transformations of finite period, Annals of Mathematics (2) 39 (1938), 127-164. MR.1503393

Department of Electrical and Systems Engineering, Washington University in St. Louis, St. Louis, Missouri 63130

Department of Mathematics, Royal Institute of Technology, 10044 Stockholm, SWEDEN 